\title{
Desarrollo Económico y Desigualdad como factores asociados a la prevalencia de la obesidad infantil en Chile
}

\author{
*Planck Barahona Urbina, Manuel Barahona Droguett
}

Universidad de Atacama, Chile

Cómo referenciar este artículo/

How to reference this article:
Barahona Urbina $\mathbf{P}$, Barahona Droguett $M$. Desarrollo Económico y Desigualdad como factores asociados a la prevalencia de la obesidad infantil en Chile. Mem. Inst. Investig. Cienc. Salud. 2019; 17(1):39-46

\begin{abstract}
RE S U M E N
El objetivo de este trabajo fue determinar los factores asociados a la prevalencia de la obesidad en párvulos a nivel nacional en el período 2001-2009 en una muestra de 553.044 párvulos. Se llevó a cabo un modelo de regresión múltiple con introducción por pasos. Como variable endógena, se utilizó la prevalencia de la obesidad en párvulos a nivel nacional. Como variables exógenas se han utilizado distintas variables macroeconómicas: la escolaridad promedio de la población (años), el Índice de Desarrollo Humano (IDH), el índice GINI, el gasto público en salud como porcentaje del PIB y el gasto en educación como porcentaje del PIB. Los resultados mostraron que las variables asociadas a la prevalencia de la obesidad infantil son el Índice de Desarrollo Humano (IDH) y el gasto público en salud. Las conclusiones del trabajo son que a medida que aumenta el Índice de Desarrollo Humano (IDH), la prevalencia en la obesidad infantil disminuye. A medida que aumenta el gasto público en salud por parte del Estado, la prevalencia de la obesidad disminuye. De los resultados del estudio se deduce la importancia que tiene el aumento del gasto público en salud en prestaciones de servicios preventivos y curativos, y en actividades de planificación familiar.
\end{abstract}

Palabras clave: obesidad, Índice de GINI, índice de desarrollo humano, gasto en salud, gasto en educación.

\section{Economic development and inequality as factors associated with the prevalence of childhood obesity in Chile}

\section{A B S T R A C T}

The objective of this work was to determine the factors associated with the prevalence of childhood obesity at national level in the period 2001-2009 in a sample of 553,044 children. A multiple regression model with step introduction was carried out. As an endogenous variable, the prevalence of obesity in preschool children at national level was used. As exogenous variables, different macroeconomic variables were used: the average schooling of the population (years), the Human Development Index (HDI), the GINI index, the public spending on health as a percentage of GDP and the spending on education as a percentage of GDP. The results showed that the variables associated with the prevalence of childhood obesity were the Human Development Index (HDI) and public health expenditure. The conclusions of this work are that as the Human Development Index (HDI) increases, the prevalence in childhood obesity decreases. As public health expenditure increases, the prevalence of obesity decreases. The results of the study show the importance of the increase in public health expenditure in preventive and curative services, and in family planning activities.

Keywords: obesity, Gini index, human development index, health expenditure, education expenditure. 


\section{INTRODUCCIÓN}

Los estudios han mostrado que la obesidad crece a niveles alarmantes en la población infantil $^{(1,2)}$. La evidencia científica ${ }^{(3)}$ ha mostrado que la prevalencia de la obesidad tiene una relación directa con el bienestar de los países medido a través del IDH (Índice de Desarrollo Humano), es decir, a medida que aumenta este índice, mayor es la prevalencia de la obesidad. Esto sugiere que la obesidad es un fenómeno común en países en vías de desarrollo y desarrollados. En un contexto global, se ha observado que en los últimos 25 Años, en América Latina la obesidad se ha multiplicado por $\operatorname{dos}^{(4)}$.

Chile se halla, también, dentro de los países con mayor sobrepeso infantil. Según un informe de la Junta Nacional de Auxilio Escolar y Becas (JUNAEB) (2016), sólo cuatro de cada diez escolares están en su peso ideal, esto es; en el año 2016 el 50,3\% de niños de prekinder presentaban sobrepeso u obesidad y en Kinder un $52 \%$. El prekinder fue el nivel de mayor aumento de la prevalencia de la obesidad y en 20 años se duplicó la tasa de obesidad en primero básico ${ }^{(5)}$.

En un estudio ${ }^{(6)}$ sobre la prevalencia de la obesidad en América Latina, se llevó a cabo un marco conceptual con el propósito de determinar los factores más comunes. El autor hace alusión al hecho de que el problema de la obesidad es multifactorial y tiene que ver con factores alimenticios de la población y socioculturales. El estudio reveló que en los estratos sociales más bajos y de menor nivel educativo se observan mayores índices de obesidad. Se observó que en las personas en situación de pobreza, la obesidad se asociaba a episodios de desnutrición en edad temprana y a factores alimentarios, socioculturales y de género. Es por tal motivo que la condición socioeconómica podría condicionar la obesidad. El mismo autor determinó que los aspectos socioculturales son relevantes a la hora de explicar la prevalencia de la obesidad en términos de su comprensión como expresión de la salud. Otros autores ${ }^{(7)}$ han puesto especial énfasis en la importancia de poner en prácticas programas de reducción de la pobreza y mejorar las condiciones de vida de la población como una manera de disminuir las desigualdades en la prevalencia de la obesidad infantil. La obesidad en este sentido es compleja y dinámica ${ }^{(8)}$.

Otros autores estudiaron ${ }^{(9)}$ la prevalencia de la obesidad en 105 países desde el año 2000 al 2006. Los países fueron agrupados por regiones: Este de Asia y Pacifico, Europa y Asia Central, Latinoamérica y el Caribe, Sur de Asia, África Subsahariana y América de Norte. El estudio utilizó la correlación de Pearson y un modelo de regresión logística múltiple. Como variables dependientes se utilizaron el Indice de desarrollo humano (IDH), el índice de GINI, el índice de democracia y la variable de disponibilidad energética. Los resultados pusieron de manifiesto que en el caso del comportamiento nutricional por Índice de masa corporal (IMC), la obesidad es mayor en países con IDH altos (niveles superiores a 0,80 ) en relación a los de ingreso medio (cuyos niveles rondan entre 0,50 y 0,80 ) y estos a su vez mayor que en los de IDH bajo (valoración inferior a 0,50). Es decir, los países con mayores IDH presentan una mayor prevalencia en obesidad. Otro hallazgo es que los países clasificados con mayor índice de democracia poseen una mayor prevalencia de obesidad de su población. En conclusión, los países clasificados con un IDH alto, los considerados como libres, así como los más equitativos en la distribución del ingreso (GINI), coinciden en tener las mayores prevalencias de exceso de peso por IMC en adultos. En una línea de investigación ${ }^{(10)}$ similar se determinó la asociación entre el Índice de Desarrollo Humano (IDH) con la diabetes tipo 2 generada por el sobrepeso y obesidad. El estudio se llevó a cabo en pacientes mayores de 20 años con prevalencia de la diabetes tipo 2 atendidos en 30 unidades de medicina familiar de la Delegación San Luis Potosí de Méjico. EI IDH del Estado de Potosí se obtuvo a través de los datos publicados por el programa de las Naciones Unidas para el Desarrollo. Para ello se utilizó una correlación de Spearman y una regresión lineal múltiple. El resultado determinó que la prevalencia de la obesidad tipo 2 puede ser explicada por el IDH como un factor socioeconómico, por lo tanto el estudio sugiere que las personas que viven en municipios con mayor IDH tienen mayor probabilidad de desarrollar la diabetes tipo 2.

En un estudio de la obesidad llevado a cabo en Chile, se encontró que en los estratos sociales más bajos, ha aumentado la ingesta de alimentos calóricos y disminuido los alimentos saludables ${ }^{(11,12)}$. En esta misma línea de investigación, se estudiaron el hábito alimenticio en escolares de los niveles socioeconómicos medio y bajo de la Región Metropolitana. Los resultados mostraron que el consumo de alimentación sana era limitado 
en ambos grupos socioeconómicos. Otro hallazgo fue que el consumo de pan era mayor en los estratos de menores ingresos y por ende de menor nivel educativo ${ }^{(13)}$.

El objetivo de este trabajo fue determinar los factores asociados a la prevalencia de la obesidad en párvulos a nivel nacional en el período 2001-2009 en una muestra de 553.044 párvulos.

\section{MATERIAL Y MÉTODOS \\ Datos y Variables}

En la elaboración de este trabajo se utilizaron datos de naturaleza agregada a nivel nacional para el período comprendido entre 2001 y 2009. Como variable endógena se consideraron los datos de la prevalencia de obesidad en párvulos a nivel nacional, proporcionado por la Junta Nacional de Jardines Infantiles (JUNJI). El número total de párvulos evaluados por año respectivamente a nivel nacional fue de: 76.831, 79.744, 83.776, 61.210, 63.166, 47.579, 48.184, 45.254, 47.300 con un total de 553.044. Como variables explicativas se utilizaron la escolaridad promedio de la población (años), datos recabados del Informe de Indicadores de la educación en Chile, el gasto en salud (como la suma del gasto público y privado en salud, abarcando la prestación de servicios de salud preventivos y curativos, las actividades de planificación familiar, las actividades de nutrición y la asistencia de emergencia designadas para la salud), el gasto público en educación como porcentaje del PIB, información proporcionada por el Banco Mundial, el Índice de Desarrollo Humano (IDH) elaborado por la PNUD (El Programa de las Naciones Unidas para el Desarrollo) y el índice de GINI, información proporcionada por el Ministerio de Planificación Nacional y Política Económica (MIDEPLAN) en base a Encuesta CASEN. Para la elaboración de este trabajo se utilizó el paquete estadístico SPSS.

\section{Análisis estadístico}

El trabajo se ha dividido en dos partes. En la primera parte se llevó a cabo un estudio de correlación de Pearson de las variables en estudio.

En la segunda, se ha estimado un modelo de regresión lineal múltiple, con la introducción de variables "por pasos" como método de estimación para lograr un buen ajuste. Como su nombre lo indica consiste en ir elaborando sucesivas ecuaciones de regresión en las que se va añadiendo cada vez un regresor más. El primer paso consiste en seleccionar el regresor de máxima correlación simple con el criterio, y se define, en consecuencia una ecuación de regresión simple con esa variable. A continuación se elabora una segunda ecuación de regresión añadiendo a la anterior otro regresor, esta vez el que más proporción de variabilidad explicada aporte sobre la ecuación anterior. Así hasta que no haya más regresores que aporten nada sustantivo, que es cuando el procedimiento acaba. Hay que indicar, y aquí esta lo destacable de este procedimiento, que en este proceso de elaboración del modelo definitivo, hay una continua reevaluación de los predictores incluidos en el modelo, de forma que si algún regresor queda explicado por los restantes (en el sentido de que carece de contribución específica propia) queda eliminado.

Formalmente el modelo de regresión se expresa de la siguiente forma:

$$
Y=\beta_{0}+\beta_{1} x_{1}+\beta_{2} x_{2}+\ldots+\beta_{k} x_{k}+\varepsilon
$$

Donde los valores estandarizados de los coeficientes $\beta_{1}, \beta_{2} \ldots \beta_{k}$ denotan la magnitud del efecto que las variables explicativas $x_{i}$ tienen sobre la variable dependiente $\mathrm{Y}$. El coeficiente $\beta_{0}$ es el término constante, y $\varepsilon$ el término error del modelo, para el que suponemos con media cero $E(\varepsilon)=0$ y varianza constante $\operatorname{Var}(\varepsilon)=\sigma^{2}, \mathrm{y}$ siendo las perturbaciones no correlacionadas $\operatorname{Cov}\left(\varepsilon_{t}, \varepsilon_{s}\right)=0$, para todo $\forall t \neq s$. 


\section{RESULTADOS}

En la Tabla 1 se puede observar que, en el caso de la variable obesidad, la media de prevalencia de obesidad infantil a lo largo del período fue de un 9,8\%. Otra de la variable que llama la atención es la escolaridad promedio de la población, es decir se observa un bajo nivel educativo de los individuos (10,9 años). Otro aspecto tiene que ver con el índice de Gini, es decir una marcada desigualdad en la distribución de la renta, lo que podría manifestarse en una mayor prevalencia de obesidad en los estratos sociales de menores ingresos. Es así que la desigualdad socava el bienestar de los individuos y está asociada a mayores índices de obesidad ${ }^{(14,15)}$. Respecto del IDH, se observa que Chile se halla dentro de los países de medio desarrollo humano de acuerdo a la clasificación de la PNUD. Respecto del gasto en educación, éste ha tenido una tendencia creciente en el período desde un 2,9\% en el año 2001 a un $4,2 \%$ en el año 2009. Igualmente sucede con el gasto en salud, $6,51 \%$ y $6,87 \%$ respectivamente. Ambos valores están por debajo el promedio de los países de la OCDE.

Tabla 1. Análisis Descriptivos de las variables en análisis. Período 2001-2009.

\begin{tabular}{lrrrrr}
\hline \multicolumn{1}{c}{ Variables } & \multicolumn{1}{c}{ Media } & \multicolumn{1}{c}{ Mediana } & \multicolumn{1}{c}{ Máximo } & \multicolumn{1}{c}{ Minimo } \\
\hline $\begin{array}{l}\text { Prevalencia de } \\
\text { Obesidad }\end{array}$ & 9,844 & 10,0 & 10,70 & 8,40 & 0,758 \\
Escolaridad promedio & 10,922 & 11,0 & 12 & 10,3 & 0,519 \\
Gasto educación \% PIB & 3,522 & 3,5 & 4,2 & 2,9 & 0,471 \\
IDH & 0,792 & 0,796 & 0,815 & 0,768 & 0,015 \\
Gasto salud \% PIB & 6,748 & 6,690 & 7,390 & 6,180 & 0,421 \\
GINI & 0,532 & 0,530 & 0,5600 & 0,510 & 0,014 \\
\hline
\end{tabular}

Fuente. Elaboración propia en bases a E-view 4. Chile se halla dentro de los países de medio desarrollo humano de acuerdo a la clasificación de la PNUD.

Luego de análisis descriptivo, la mejor forma de determinar una relación entre las variables es haciendo uso de la metodología de Correlación de Pearson. De los resultados de la Tabla 2 podemos observar correlaciones positivas y negativas entre las variables. En cuanto a las interpretaciones podemos decir que, en el caso de las variables obesidad y escolaridad se observa una correlación negativa, lo que nos está indicando que a medida que disminuye la escolaridad de los individuos o de las familias aumenta la prevalencia de la obesidad en la población infantil. Este resultado es consistente con la evidencia científica en la que se ha encontrado que la obesidad se da con mayor fuerza en los estratos económicos más bajos y de menor escolaridad. Respecto de la relación entre obesidad y gasto en educación realizada por el gobierno se puede observar una asociación igualmente negativa, es decir, a medida que aumenta el gasto público en educación la prevalencia en la obesidad disminuye. Esto nos estaría indicando que la educación, entendida como un aumento del capital cultural de los individuos, es un elemento importante en la formación de los hábitos alimenticios en los primeros años de vida de los niños. En cuanto a las variables obesidad y el índice de desarrollo humano (IDH) se puede observar que con un aumento en este índice disminuye la prevalencia de la obesidad. Este resultado es consistente con la evidencia internacional de países con un medio desarrollo humano. Se encontró que el gasto en salud de parte del Estado tiende a disminuir la prevalencia d la obesidad. Por otro lado, se halló que la desigualdad de los ingresos (GINI) reduce el bienestar medido a través del IDH. Este resultado es consistente con la evidencia internacional en la cual se ha encontrado una relación inversa entre indicadores de desigualdad con el indicador de calidad de vida ${ }^{(16,17)}$. Respecto de los valores de matriz de correlación, se observan asociaciones leves lo que nos hace suponer la no existencia de multicolinealidad entre las variables. 
Tabla 2: Matriz de Correlación de Pearson de las variables en análisis.

\begin{tabular}{|c|c|c|c|c|c|c|}
\hline & Obesidad & Escolaridad & $\begin{array}{l}\text { Gasto Educ } \\
\text { (\% PIB) }\end{array}$ & IDH & $\begin{array}{r}\text { Gasto salud } \\
(\% \text { \% }) \\
\end{array}$ & GINI \\
\hline Obesidad & 1,000 & $-0,889 *$ & $-0,496$ & $-0,890 *$ & $-0,547$ & 0,313 \\
\hline Escolaridad & $-0,889 *$ & 1,00 & 0,34 & $0,93 *$ & 0,29 & $-0,30$ \\
\hline $\begin{array}{l}\text { Gasto Educ } \\
\text { (\%) PIB) }\end{array}$ & $-0,496$ & 0,34 & 1,00 & 0,25 & $0,72 * *$ & 0,08 \\
\hline IDH & $-0,890 *$ & 0,93 & 0,25 & 1,00 & 0,23 & $-0,31$ \\
\hline $\begin{array}{l}\text { Gasto salud } \\
\text { (\%)PIB }\end{array}$ & 0,547 & 0,29 & $\begin{array}{r}0,72 * \\
*\end{array}$ & $\begin{array}{r}0, \\
23\end{array}$ & 1,00 & 0,08 \\
\hline GINI & $\begin{array}{r}0,3 \\
13\end{array}$ & $-0,30$ & 0,08 & $0,3 \overline{1}$ & $-0,08$ & 1,00 \\
\hline
\end{tabular}

Fuente: Elaboración propia en bases a resultados E-view 4.0. Significancia estadística $* \mathrm{p}<1 \%, \mathrm{p} * *<5 \%$.

\section{Resultados modelo econométrico}

De acuerdo a los modelos de la Tabla 3 podemos observar los resultados de la Regresión múltiple con introducción por pasos. De las regresiones propuestas, el modelo 11 es el que mejor cumple con las condiciones de validez de un modelo. Se puede observar una buena significatividad de las variables. Las variables estarían explicando el 91 por ciento de la prevalencia de la obesidad infantil. Se observa un $p$-valor pequeño lo que indica la aceptación de la hipótesis de la significatividad conjunta de todos los parámetros del modelo. Por otro lado el valor el estadístico Durbin-Watson no está demasiado lejos de 2, esto estaría indicando que los problemas de auto correlación no son relevantes. Respecto a los resultados del modelo se encontró que las variables IDH y el gasto público en salud, tienen una relación inversamente proporcional con la variable endógena, es decir un aumento de estas dos variables disminuye la prevalencia de la obesidad infantil.

Tabla 3. Regresión múltiple con Introducción por pasos. Serie 2001-2009. Variable dependiente Prevalencia de Párvulos a nivel nacional.

\begin{tabular}{lrr}
\hline \multicolumn{2}{l}{ Modelos } & \multicolumn{1}{l}{ Coeficientes } \\
\hline Modelo 1 & 35,400 & 0,0228 \\
\hline Constante & $-0,6604$ & 0,3707 \\
Escolaridad & $-23,246$ & 0,3533 \\
IDH & 0,82 & \\
R2 & 1,62 & \\
Durbin-Watson stat & 0,005830 & \\
Prob(F-statistic) & & \\
Modelo 2 & 15,821 & 0,0111 \\
\hline Constante & $-0,3393$ & 0,6812 \\
Gasto en educación \%PIB & $-0,708$ & 0,4507 \\
Gasto salud \% PIB & 0,32 & \\
R2 & 3,12 & \\
Durbin-Watson stat & 0,005571 & \\
Prob(F-statistic) & & \\
Modelo 3 & 3,1332 & 0,7366 \\
\hline Constante & $-0,8458$ & 0,1567 \\
Gasto en educación \%PIB & 18,2075 & 0,3153 \\
GINI & 0,37 & \\
R2 & 0,7960 & \\
Durbin-Watson stat & & \\
Prob(F-statistic) & 0,247683 & \\
Modelo 4 & & \\
\hline Constante & 8,8568 & 0,4090 \\
Gasto salud \% PIB & $-0,9435$ & 0,1572 \\
GINI & $-0,1382$ & 0,4377 \\
R2 & 0,37 & \\
Durbin-Watson stat & 0,310 & \\
Prob(F-statistic & & \\
Modelo 5 & 0,248417 & \\
\hline
\end{tabular}




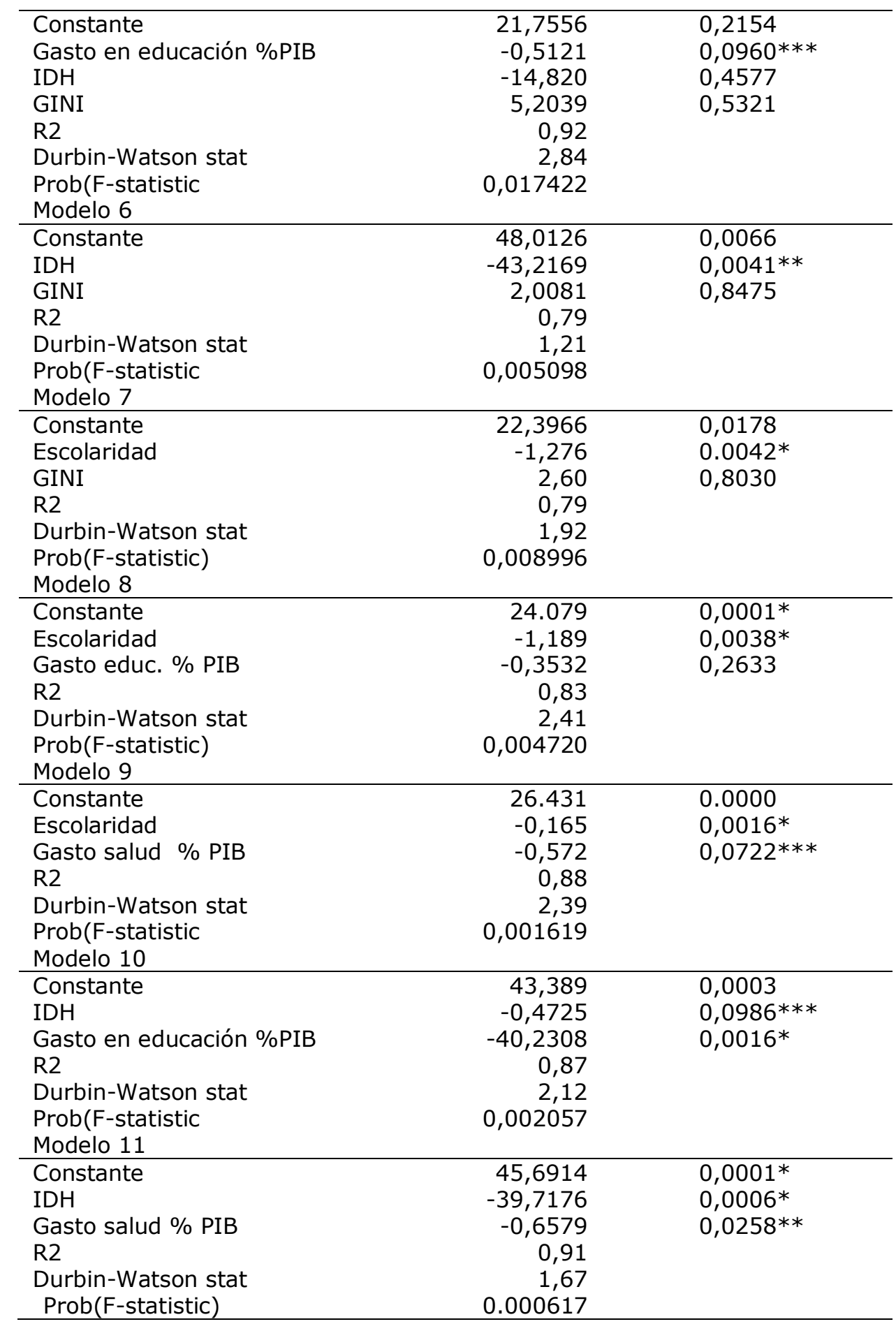

Fuente: Elaboración propia. En bases a resultados E-view 4.0. Significancia estadística *p<1\%, $\mathrm{p}^{* *}<5 \%, \mathrm{p}^{* * * *}<10 \%$,

\section{DISCUSIÓN}

El objetivo de este trabajo de investigación fue determinar los factores asociados a la prevalencia de la obesidad infantil para una muestra de 533044 niños para el período 2001-2009. En una primera etapa del análisis se pudo observar la existencia de una relación negativa entre las variables prevalencia de la obesidad y escolaridad, es decir, a medida que disminuye la escolaridad de los individuos o las familias, aumenta la prevalencia de la obesidad en la población infantil( ${ }^{(18)}$. Este resultado es consistente con un estudio llevado cabo a 144 países desarrollados y en vías de desarrollo, cuyo objetivo fue observar las tendencias del sobrepeso y la obesidad en niños en edad preescolar. El estudio analizó un total de 450 encuestas transversales representativas. El estudio concluyó que 43 millones de niños (35 millones en países en desarrollo) tenían sobrepeso y eran obesos; 92 millones estaban en riesgo de sobrepeso. El mismo estudio halló que la prevalencia mundial 
de sobrepeso y obesidad infantil aumentó del 4,2\% en 1990 al 6,7\% en 2010 y se espera que esta tendencia alcance el 9,1\% (aproximadamente 60 millones) en 2020. Por otro lado, la prevalencia estimada de sobrepeso y obesidad infantil en África en el año 2010 fue del $8.5 \%$ y se espera que alcance el $12.7 \%$ en 2020 . Sin embargo, se observó que la prevalencia es más baja en Asia que en África (4.9\% en 2010), pero el número de niños afectados (18 millones) es más alto en Asia. Es decir, la evidencia empírica ha puesto de manifiesto que la obesidad en las edades tempranas no sólo está estrechamente relacionada con factores alimentarios, sino también con factores que tienen que ver con el nivel socioeconómico de las familias, su nivel educativo y con su condición de pobreza.

Respecto de la relación entre obesidad y el gasto público en educación que llevan a cabo los gobiernos, se pudo observar que un aumento del gasto en esta área, la prevalencia de la obesidad disminuye. La labor del Estado es procurar un sistema educativo público que mejore el capital humano de los individuos de una sociedad. Es así, que la educación ha sido un elemento vital para el desarrollo y que influye en la compresión de lo beneficioso que resultan los buenos hábitos alimenticios. Por otra parte, el resultado de la correlación entre el IDH y la prevalencia de la obesidad se halló que es consistente con la evidencia internacional, en cuanto que países con un medio desarrollo humanos tienen menor prevalencia de obesidad respecto de países con un alto desarrollo humano.

Respecto a los resultados del modelo econométrico, se encontró que, ceteris paribus, un aumento en un punto porcentual en la variable IDH (Índice de desarrollo humano) la prevalencia en la obesidad infantil disminuye en 39,7 puntos porcentuales. Con un aumento en el gasto en salud en un punto porcentual, la prevalencia de la obesidad disminuye en 0,6579 puntos porcentuales. En este sentido, los resultados del estudio han puesto de manifiesto la importancia que tiene el aumento del gasto público en salud en prestaciones de servicios preventivos y curativos, y de actividades de planificación familiar, con el propósito de mejorar la nutrición en las edades tempranas de la niñez. A la larga, las inversiones en el cuidado de la salud benefician directamente el crecimiento económico del país.

Las limitaciones del trabajo tienen que ver con el acceso a una base de naturaleza desagregada, esto permitiría ampliar la línea de investigación y enriquecer el modelo.

\section{AGRADECIMIENTOS}

Agradecemos a la Junta Nacional de Jardines Infantiles (JUNJI) por habernos proporcionados la base de datos que nos permitió elaborar este trabajo de investigación. Especialmente a la Abogada Jannina Osorio Carmona, por sus amables gestiones.

Financiación: El trabajo fue financiado con fondos propios de los autores.

Conflicto de interés: Los autores no declaran conflictos de intereses.

\section{REFERENCIAS BIBLIOGRAFIA}

1. Atalah E. Epidemiology of obesity in Chile, clinical medical journal. 2012; 23(2): 117123.

2. Janssen I, Shields M, Craig C, Tremblay $M$. Prevalence and secular changes in abdominal obesity in Canadian adolescents and adults 1981 to 20072009. Obes Rev. 2011; 12: 397-405.

3. Overview Human Development Report. Human Development For Everyone. PNUD. 2016; 1-40.

4. Peña M, Bacallao J. Obesity in poverty: an emerging problem in the Americas, Futures Magazine. México. 2006; 3 (10): 103-117.

5. Nutritional map report 2016. JUNAEB. 2017; 1-29

6. Figueroa P. Obesity and Poverty: conceptual framework for its analysis in Latin America, Saude Soc. Sao Paulo. 2009; 18 (1): 103-117.
7. Robinson $L$, Siles $M$, Schmidt A. Social capital and the reduction of poverty: towards a paradigm. Social capital and poverty reduction in Latin America and the Caribbean: in search of a new paradigm. Santiago, Chile: CEPAL, 2003; 51-114.

8. Peña M, Bacallao J. Obesity in poverty: an emerging problem in the Americas, Futures Magazine. México. 2006; 3 (10): 103-117.

9. González I, Estrada A, Álvarez C, Serra L. Excess weight, economic, political and social aspects in the world: an ecological analysis. Cad. Saude Publica. 2011; 27 (9): 1746-1756.

10. Mendoza M, Games G, Salas P, Ortiz J, Ramírez M, Salas J. Association of the human development index and diabetes mellitus type 2 in units of family medicine of the state San Luis Potosí, Méjico. Aten Fam. 2017; 24 (4): 156-159. 
11. Atalah E. Epidemiology of obesity in Chile. Rev. Med. Clin. Condes. 2011; 23 (2): 117-123.

12. Crovetto $M$, Uauy $R$. Changes in the apparent consumption of nutrients in Santiago 1988-1997 in households according to income and their probable relationship with the pattern of chronic non communicable diseases. Medical magazine. 2010; 138: 1091-1108.

13. Olivares S, Vio F. Comparative study of the diet of Chileans with the Mediterranean diet. Chilean Magazine of Nutrition. 2010; 28 (2): 269-276.

14. De Onis M, Blössner M, Borghi E. Global prevalence and trends ofoverweight and obesity among preschool children. Am J Clin Nutr. 2010; 92: 1257-64.
15. Wilkinson, R. Pickett, K. The Spirit Level. Why More Equal Societies Almost Always Do Better. London: Penguin. (2009b).

16. Oishi, S, Kesebir, S, Diener, E. Income inequality and happiness. Psychological Science, 2011; 22(9): 1095-1100.

17. Diener, E., Diener, M., \& Diener, C. Factors predicting the subjective wellbeing of nations. Journal of Personality and Social Psychology, (1995). 69(5): 851-864.

18. Díaz M, Rocío R. Obesity: prevalence and relationship with educational level in Spain, Nutr. clín. diet. hosp. 2016; 36(3): 181-188. 